\title{
Forecasting the influence of vehicle columns with liquid cargoes on bearing systems in agriculture
}

\author{
Sergey Gridnev ${ }^{1}$, Alexey Budkovoy ${ }^{1}$, Yuriy Skalko ${ }^{2, *}$, and Rinat Muchtarov ${ }^{1}$ \\ ${ }^{1}$ Voronezh State Technical University", 14, Moskovskiy prospect, 394026, Voronezh, Russia \\ ${ }^{2}$ Moscow Institute of Physics and Technology, 9, Institutskiy per., 141700, Dolgoprudny, Moscow \\ Region, Russia
}

\begin{abstract}
Studies have been performed to evaluate the dynamic effect of columns of the same type of agricultural tanker truck on the beam-bearing systems of transport structures during movement in braking mode. The design scheme of the general oscillatory system is selected, a mathematical and numerical model of its behavior is developed, an algorithm for joint nonlinear oscillations is constructed. On the basis of the developed expert models, a computer complex was created using the simulation package of dynamic and event-driven systems Simulink MATLAB systems. Using it, a series of computational experiments was carried out to simulate situations of passage of convoys of cars with different parameters and with different driving modes. Various parameters varied: the speed of the column, the distance between the cars, the speed at the time of the start of braking, the place where the first car began to brake, the amount of steady deceleration, the number of tank compartments, and the value of operational underfilling. Based on the analysis of the results of numerical studies, the features of the dynamic effect of vehicles convoys carrying liquid cargo on load-bearing systems from the standpoint of the emergence of excess dynamic pressure on transport facilities and the need for its consideration are shown. Cases of a dangerous combination of parameters and driving modes are highlighted, and the need to distinguish such columns in a special class of moving load is shown. A significant increase in the dynamic coefficients of deflections in comparison with solid loads is shown. The need for full-scale research in this area with the aim of introducing amendments to regulatory documents has been proved.
\end{abstract}

\section{Introduction}

The globalization of the world economy and the favorable geographical location of Russia at the intersection of the largest trade routes of the Eurasian continent allow our country to increase its trade with the leading countries of this region every year, which is impossible without the corresponding development of the road transport network. The growing volumes of cargo transportation bring the economy of individual regions to a

\footnotetext{
*Corresponding author: skalko@mail.mipt.ru
} 
qualitatively new level, while at the same time increasing the requirements for transport infrastructure. It is obvious that under such conditions it is necessary to adequately predict the stress-strain state (VAT) of newly designed and reconstructed objects, as well as to reliably evaluate the reliability and residual life of the operating load-bearing structures of transport structures. Particular attention should be paid to assessing the possibility of passing a convoy of vehicles over existing structures in emergency situations and for the needs of the armed forces of the Russian Federation.

Strength analysis of any structure begins with the determination of the loads perceived by the structure and working conditions. Unfortunately, the current regulatory documents adopted a rather simplified description of moving loads, which forms an engineer only a superficial understanding of the complex processes that underlie and lay down the fundamental principles of the dynamics of structures. All this prompted the authors to the need for a deeper study of such dynamic effects that are as close as possible to the actual operating conditions and are most dangerous from the point of view of the occurrence of excess impact on transport facilities. Obviously, such effects are unsteady and short in time.

Among the moving loads, vehicles transporting liquid cargoes (tankers) in tank cars are of particular importance. These multilevel dynamic systems themselves are capable of causing complex oscillatory processes under conditions of unsteady influences, such as, for example, emergency braking. Currently, research trends in tank truck dynamics cover mainly the issues of their safe operation (stability against tipping over [1], load and strength of individual components and structures [2,3], damping of vibrations of liquid cargo [4,5], etc.), improving the design of the tank in terms of reducing material consumption [4,5], as well as optimizing the parameters of the volume of transported liquid in terms of economic costs.

A deep generalization was achieved by the authors of [6]. It describes in detail current advances in the field of fluid dynamics in terms of solving applied problems. Free and forced vibrations of a free surface, equivalent mechanical models, methods of damping fluid vibrations, and its parameters are described. The dynamics of bodies with cavities containing liquid during its interaction with elastic systems, splashing in road containers and ships, water hammer, oscillations in a weak gravitational field, and numerical methods for solving problems are considered separately. Among recent foreign publications, the study [7] is devoted to the study of uneven motion along a beam on an elastic foundation; fluid oscillations in railway tanks with their partial filling under conditions of sharp braking are considered in [8]. Joint vibrations of the "vehicle-bridge" system were thoroughly studied in [9], the potential energy of deformation of the span, its dynamic response is analyzed, vibration resistance and dynamic coefficients are estimated. The problems of the influence of road surface roughness on the dynamic load of a bridge from a column of vehicles in a probabilistic setting were considered in [10]. However, none of these works solves the problem of joint oscillations of a multilevel liquid-vehicle-span dynamic system with the possibility of taking into account the microprofile. carriageway in a single computing space. The issues of the dynamic impact of vehicles with liquid loads as a single moving load, or as part of a column, are not studied, the dynamic coefficients of deflections for this case are not estimated.

The main feature of the studies performed by the authors should be emphasized on the specifics of the dynamic effects of such complex oscillatory systems as tank trucks without considering the strength of their components, the possibility of tipping over, traffic safety and other issues that do not go beyond the scope of the research object itself. The main goal in this case is the normalization of the dynamic impact for subsequent widespread use in the calculations and design of transport structures for dynamic moving load in a simplified setting [7], or a deeper study of the VAT structures. The task is to identify the main factors 
of the dynamic impact of vehicles with liquid cargoes and predict the behavior of loadbearing structures under such loads.

\section{Materials and methods}

The main objectives of the study include:

- the choice of the design scheme of the oscillatory system with the necessary and sufficient degree of its discretization;

- development of mathematical and numerical models of the behavior of such a system under unsteady conditions;

- the construction of an algorithm for calculating joint nonlinear vibrations of loadbearing systems of transport structures and a column of tankers during braking and acceleration;

- the construction of expert models within the framework of a single computer complex and the implementation of numerical studies to adequately assess the nature of the dynamic effects and the values of the dynamic coefficients of the moving load.

A column formed using a basic flat nonlinear dynamic model of a tank truck as a car with five degrees of freedom (used to study movement at a constant speed), moves at a constant speed, the same interval and the start of braking of the first car at the moment of entry, mid-passage or exit (fig. 1)

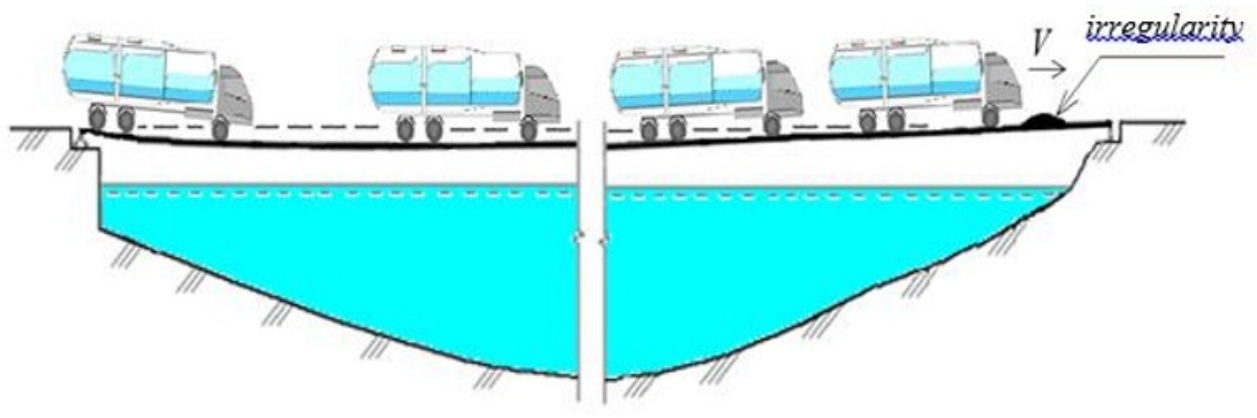

Fig. 1. The design scheme of the task.
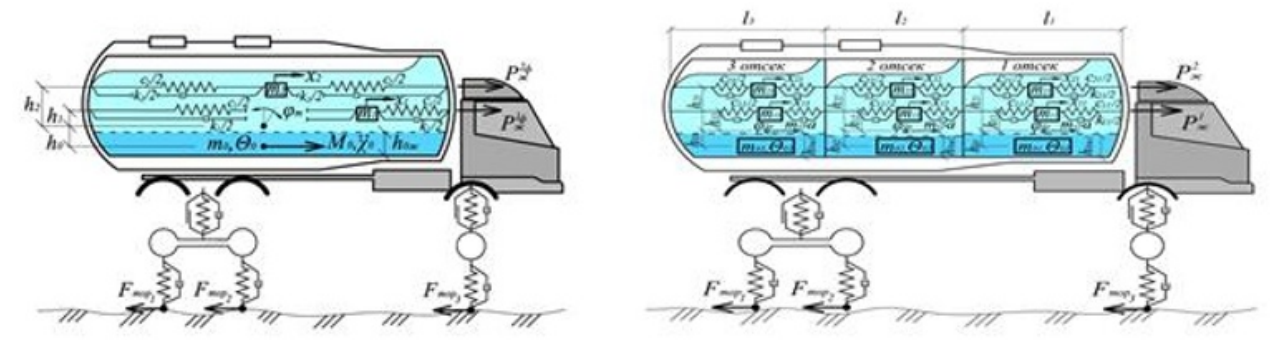

Fig. 2. Flat dynamic model of a tank truck (system with 5 degrees of freedom).

The longitudinal vibrations of the liquid in the tank were taken into account using the well-known mechanical model, in which the liquid is replaced by an equivalent solid, and the force of interaction of the liquid with the capacity is directly proportional to the relative displacement of the named body (in the absence of damping). Moreover, it is believed that the part of mass $\mathrm{m} 0$ located in the lower region of the tank does not participate in oscillations with respect to the tank reservoir (fig. 2). 
When creating the model, the case of fluid oscillations of mass $m_{\mathrm{fl}}$ and density $\rho_{f l}$ was considered in a parallelepiped-shaped vessel with dimensions $l \times b \times h_{\text {pot }}$. If the height of the liquid level is $\mathrm{h}$, then the mass of the liquid $\mathrm{mn}$ participating in the oscillations in the form with number $\mathrm{n}$ and frequency $\omega \mathrm{n}$, the corresponding stiffness coefficient $\mathrm{cn}$, and the distance hn are determined by formulas (1).

$$
\begin{gathered}
\omega_{\mathrm{n}}=\sqrt{(2 n-1) \pi(g / l) t h\left[\pi(2 n-1) \frac{h}{l}\right]} \quad m_{\mathrm{n}}=m_{\mathrm{q}} \cdot 8 \frac{l}{h} \frac{t h[(2 n-1) \pi-1}{(2 n-1)^{3} \pi^{3}} \\
c_{\mathrm{n}}=m_{\mathrm{q}} \cdot 8 \frac{g}{h} \frac{t h^{2}\left[(2 n-1) \pi \frac{h}{l}\right]}{(2 n-1)^{2} \pi^{2}} \quad h_{\mathrm{n}}=\frac{h}{2}\left(1-\frac{l}{h} \frac{\left.t h(2 n-1) \pi \frac{h}{2 l}\right]}{(2 n-1) \pi}\right) \\
m_{n}\left(\ddot{x}_{n}+h_{n} \ddot{\varphi}\right)+k_{n} \dot{x}_{n}+c_{n} x_{n}-m_{n} g \varphi-m_{n} a=0
\end{gathered}
$$

where $\varphi$ - angle of rotation of the body (mechanical part) of the tank truck.

A.O. Shimanovsky proposed the introduction of an exponential relationship for accounting for hydroblow [12]. Then the stiffness used in the mechanical model is as follows:

$$
c_{\mathrm{n}}^{\prime}=\left(\begin{array}{l}
c_{n}, x_{n} \leq x_{n}^{0} \\
c_{n} \cdot \exp \left(\frac{x_{n}-x_{n}^{0}}{3\left(x_{n}^{\max }-x_{n}\right.}\right), x_{n}>x_{n}^{0}
\end{array}\right.
$$

where $x_{n}^{0}$-displacement of the $n$th load when the ceiling is soaked;

$x_{n}^{\max }-$ maximum possible shift of the nth load.

Graphically, this relationship is as follows (fig. 3).

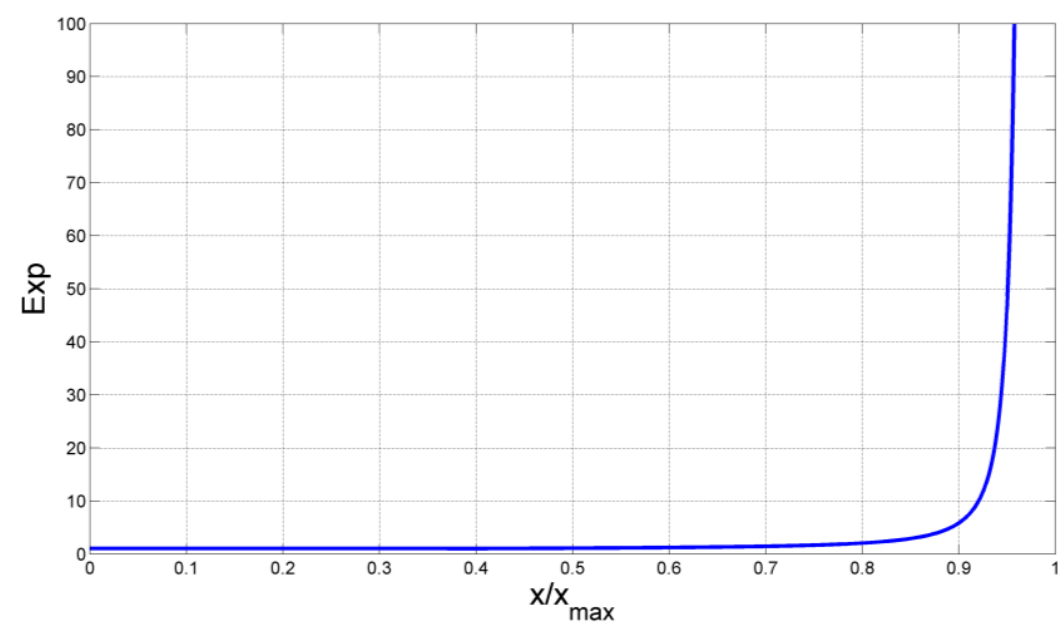

Fig. 3. The graph of the exponential relationship for accounting for water hammer. 
The basic equations of motion of the carrier system are described in [13].

The main factor determining the dynamic effect of a single moving load under conditions of equal alternating motion is the value of the steady-state acceleration. If we are talking about emergency braking on the span, then the place of braking and the length of the section, within which the speed of movement sharply changes, should be among the determining factors. In the case of vehicles moving in a convoy, the distances between cars in the convoy and the initial braking speed should be added to them [13]. Of course, this is true for both solid and liquid cargoes. However, in the case of the movement of tankers, the internal parameters of the body with the liquid become very important, among which it is necessary to highlight the length of its free surface, that is, the presence of compartments, the level of filling and the damping properties of the liquid. The work of the authors [14] is devoted to the study of the effects of single tank trucks on beam structures in a flat setting.

\section{Results}

To obtain numerical data, a series of computational experiments was carried out to simulate the braking of a convoy of three triaxial tank trucks 66052 based on KamAZ with a mass of 24 tons on a steel-reinforced concrete span with a length of $42.5 \mathrm{~m}$ in the event of an emergency stop when the head vehicle leaves the bridge. To simplify the task, we consider tankers to be equally filled and with the same number of compartments. Braking with an initial speed of $20 \mathrm{~m} / \mathrm{s}$ was considered. The range of accelerations being researched on is 2 $\ldots 6 \mathrm{~m} / \mathrm{s} 2$. For modeling, we used an improved computing system based on the MATLAB system, which was previously developed by the authors in the simulation package for dynamic and event-driven systems Simulink.

The need to allocate tankers in a separate class of loads is clearly illustrated in fig. 4. Maximum deflections at extreme fill levels depending on the number of compartments. Acceleration of $6 \mathrm{~m} / \mathrm{s} 2$, distance $-20 \mathrm{~m}$, the beginning of braking within the middle of the span. For comparison, a graph for a solid load is given. In the case of a solid cargo and a tank with three compartments, the oscillation schedules are quite close, which can be regarded as one of the most effective ways to damp fluid oscillations in the cavity

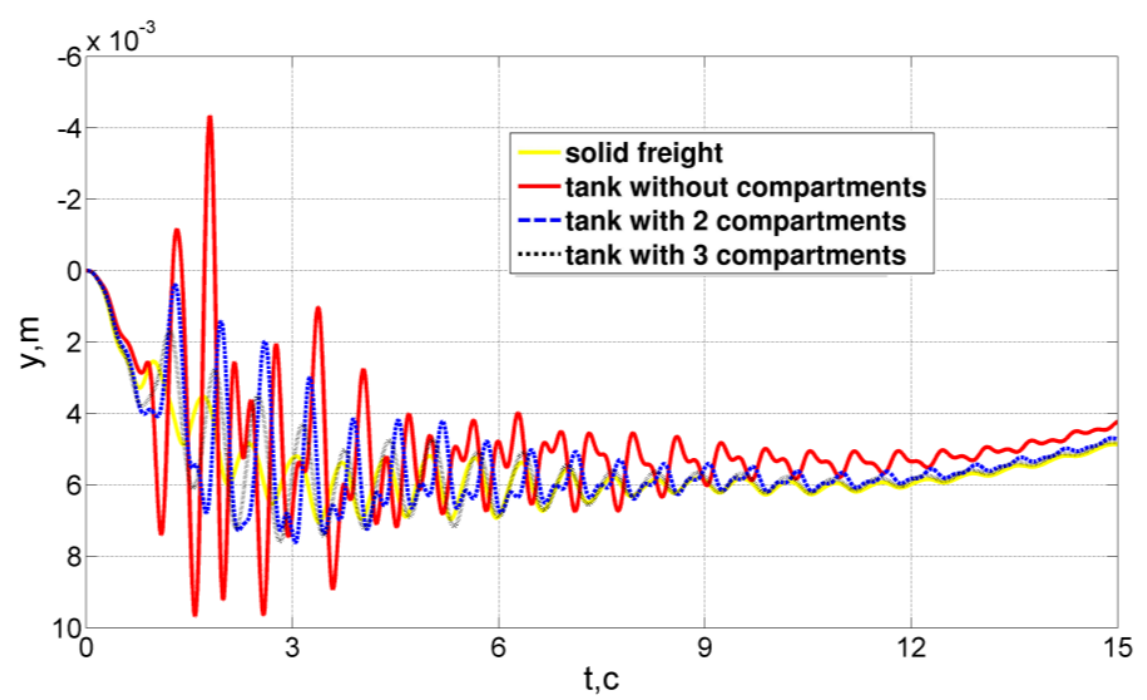

Fig. 4. Dynamic deflection of the middle section of the span with a different design of the tank cavity and the extreme level of filling. 
The effect of acceleration on dynamic deflections is shown in fig. 5. Distance $20 \mathrm{~m}$, number of compartments - 2, filling level $95 \%$.

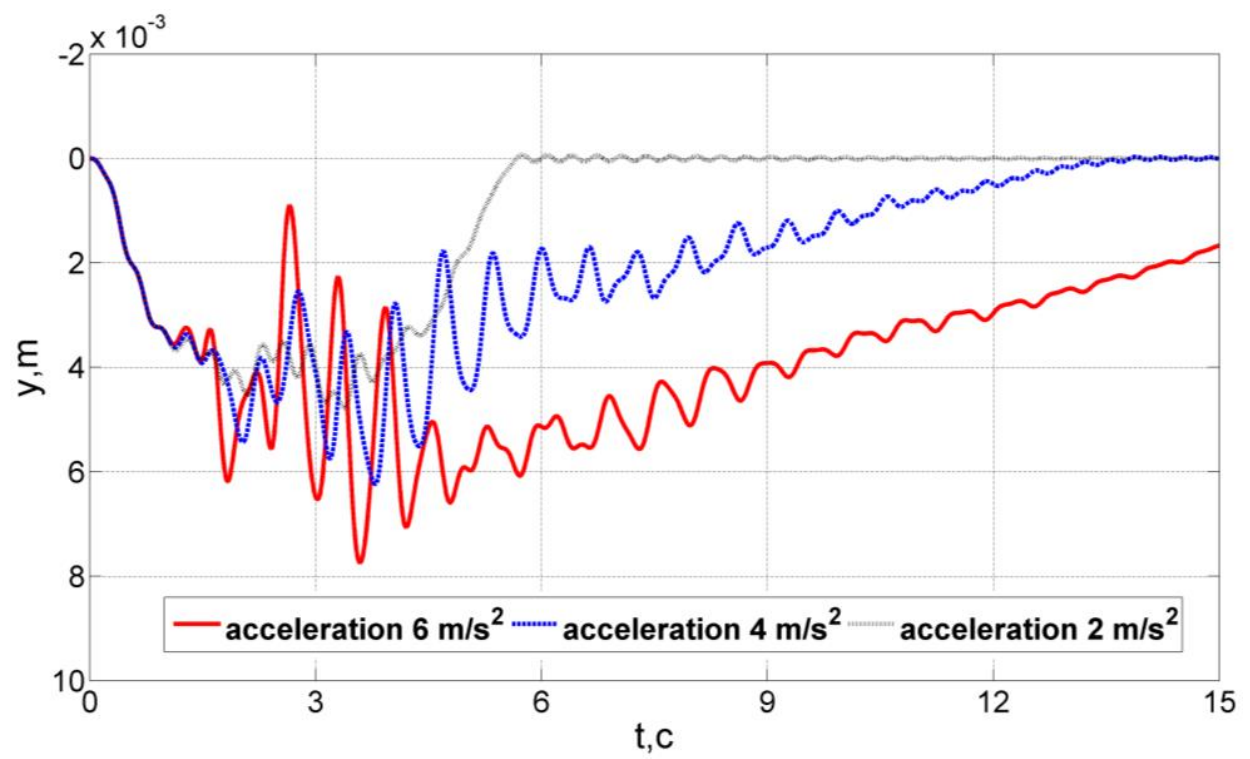

Fig. 5. Dynamic deflection of the middle section of the superstructure at various accelerations.

The characteristic nonlinear relationship between the dynamic deflections and design features and filling level is well illustrated in the graphs of the total dynamic axial pressure (fig. 6). The fill level is selected extreme for each number of compartments: without compartments - 85\%, $2-96 \%, 3-98 \%$. The distance between the tanks is $20 \mathrm{~m}$, the acceleration is $6 \mathrm{~m} / \mathrm{s}^{2}$, the beginning of braking in the middle of the span. The graphs show the difference in magnitude, frequency and nature of the impact in time with the appearance and absence of acceleration.

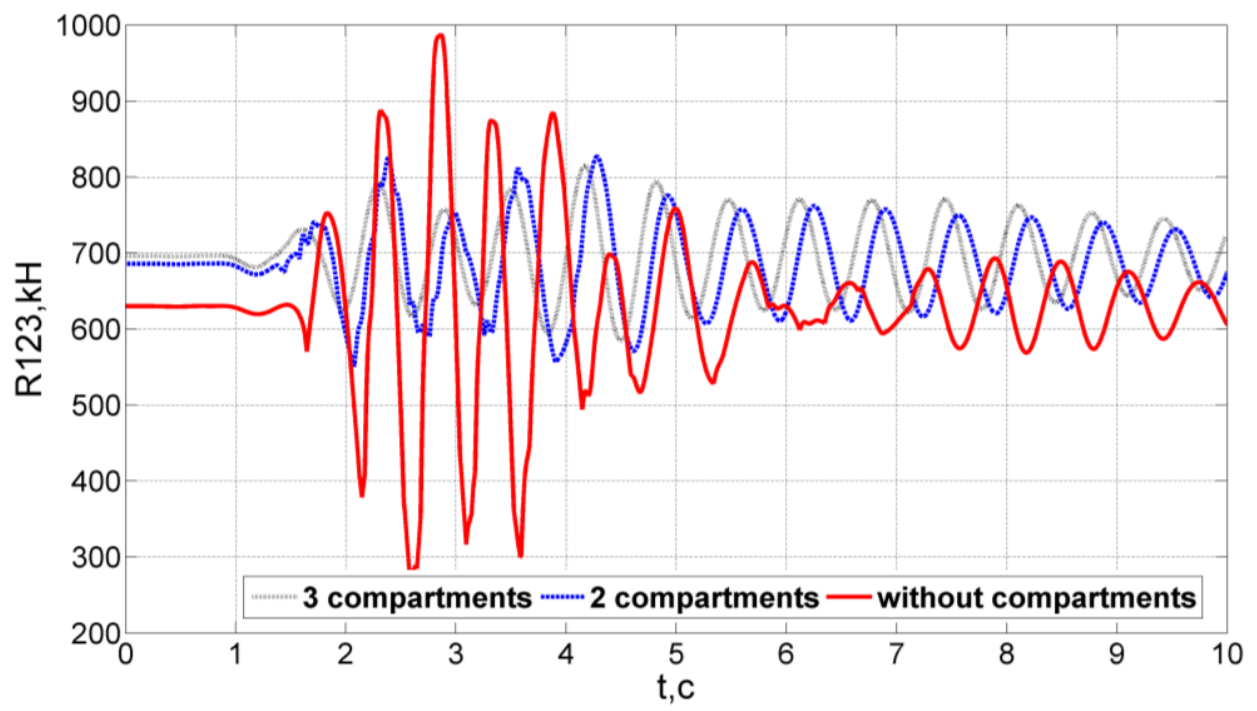

Fig. 6. The total dynamic pressure from the column of tankers with different cavity design and extreme fill level. 
Let us further consider the influence of the braking area in relation to the number of compartments at a standard distance of $20 \mathrm{~m}$ and acceleration of $6 \mathrm{~m} / \mathrm{s}^{2}$ (fig. 7).
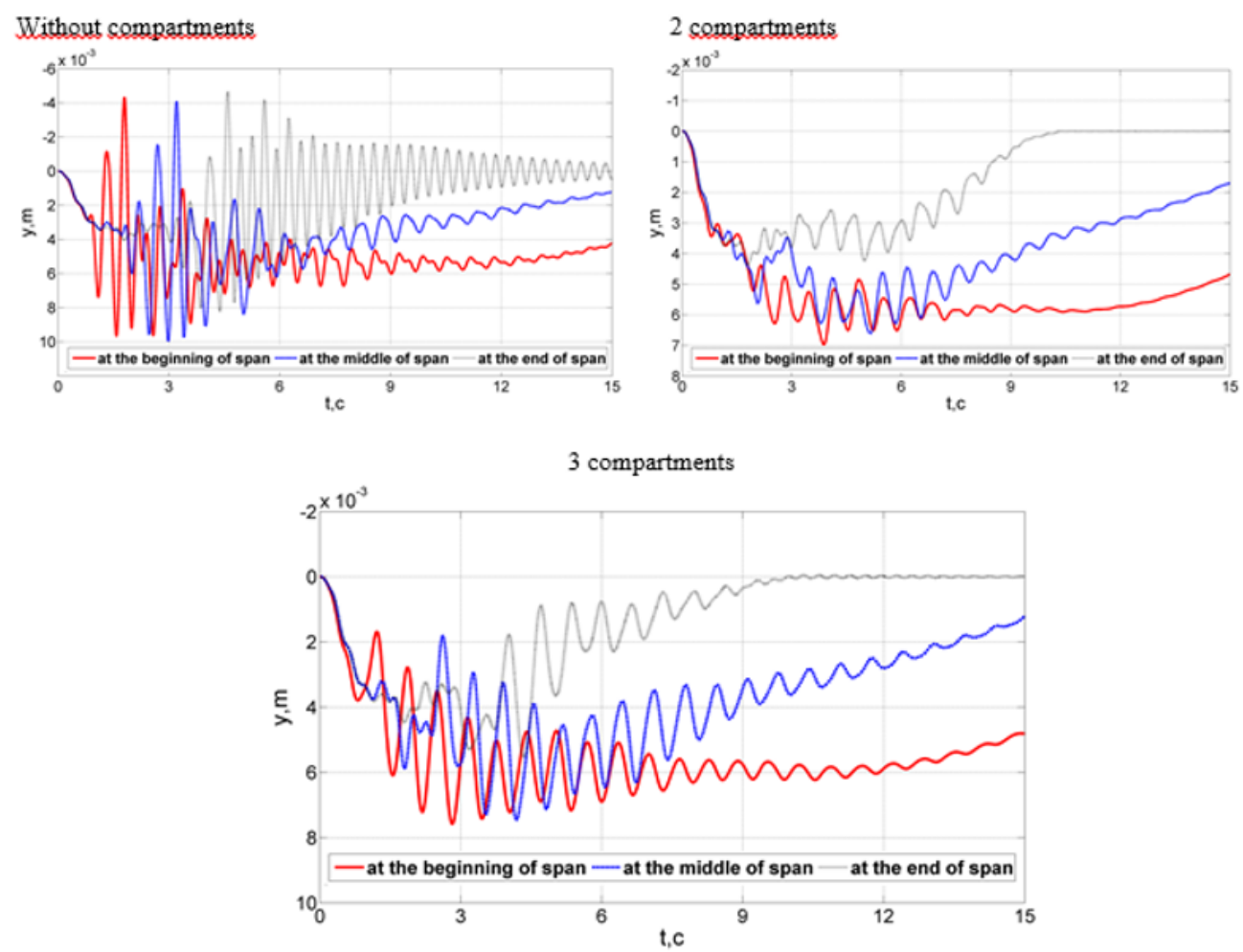

Fig. 7. Graphs of dynamic deflections in relation to the place of braking and cavity design.

As seen from the graphs, the maximum dynamic deflections during braking at the beginning and middle of the span are quite close and differ only in the time of their achievement. The dynamic pressure during braking at the end of the span is much lower.

We will show how the decrease in the distance between tankers below the standard affects the dynamic deflection when braking a tank with two compartments at $95 \%$ filling in different parts of the span (fig. 8). 

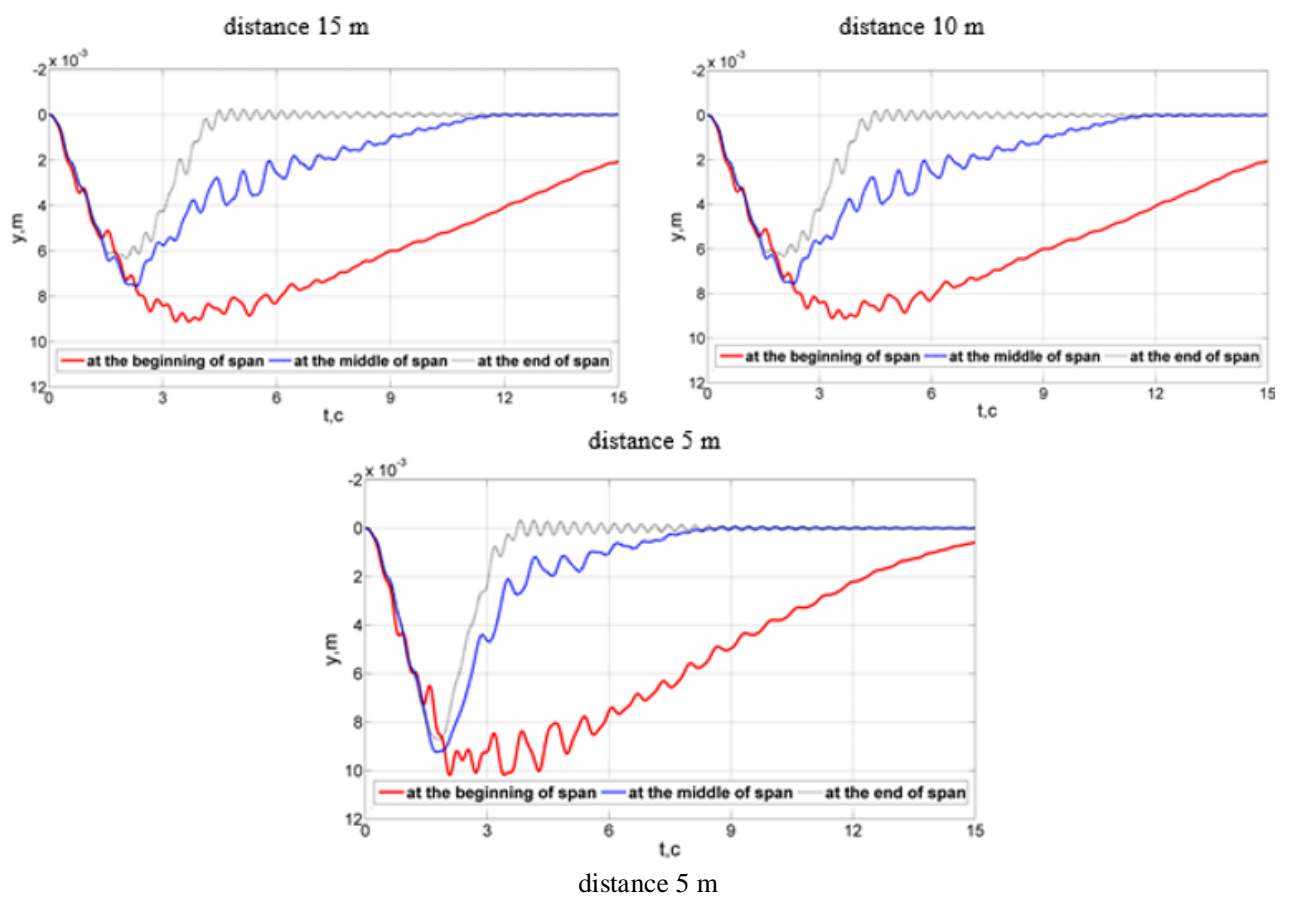

Fig. 8. The graph of the dynamic deflections of the middle section with a decrease in the distance between the tanks during braking in different parts of the span.

Obviously, with a decrease in the distance between the tanks, the maximum deflection increases. In this case, the difference between the maximum values with decreasing distance decreases.

The maximum dynamic coefficients in relation to the number of compartments and the distance between tankers in the column can be represented graphically (fig. 9)

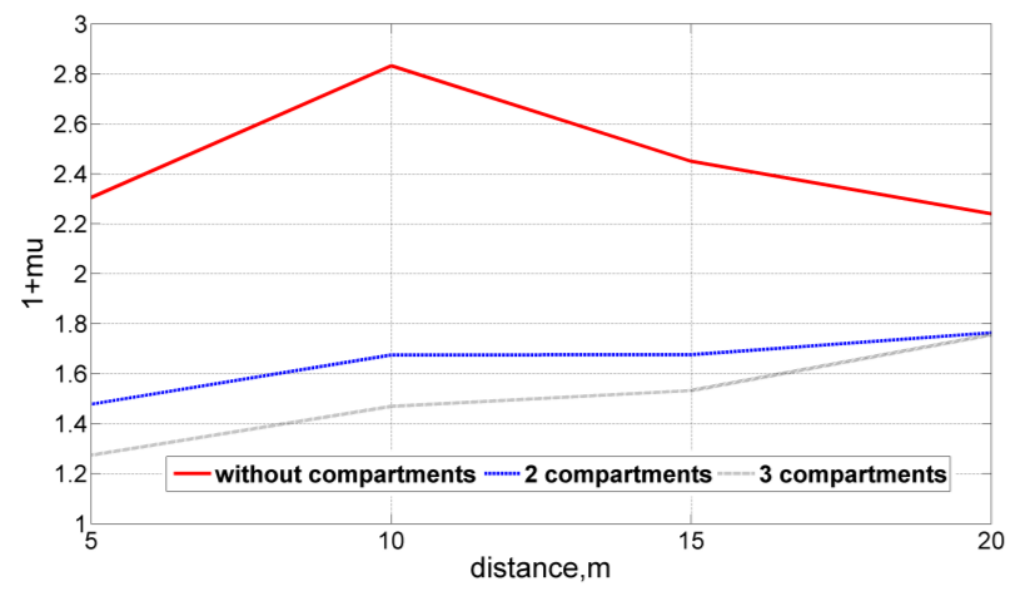

Fig. 9. The maximum dynamic coefficients in relation to the number of compartments and the distance between tankers in the column.

The decrease in dynamic coefficients with decreasing distance is associated with an increase in the static value of the deflection due to the approach of the resultant forces of 
dynamic pressure from each tank truck to each other. The absolute value of the maximum dynamic deflection with decreasing distance is steadily increasing.

\section{Discussion}

The main findings from the results of numerical studies include the following points:

1. The results of numerical studies show that the dynamic coefficients for the deflections of the middle section from the dynamic effects of the braking column of tank trucks significantly exceed the standard values;

2. Significantly, the dynamic impact is exerted by the interval between the column cars, the value of operational underfilling, the amount of braking acceleration and, to a lesser extent, the braking start speed;

3. Acceleration of braking significantly affects the amplitudes of oscillations and causes a surge in high-frequency oscillations with large amplitudes at the beginning of braking, especially in the absence of compartments;

4. With a decrease in the distance between cars, the values of the deflections begin to grow and reach a maximum value at an extreme distance of $5 \mathrm{~m}$;

5. An increase in the initial braking speed reduces the oscillation frequency and slightly reduces the amplitudes.

\section{Conclusions}

1. In carrying out the research, theoretical and methodological foundations of numerical simulation of the passage of columns of specialized vehicles with liquid cargo on loadbearing systems were developed

2. Analysis on the results of numerical studies and their verification allows us to formulate recommendations for revising the regulatory framework of dynamic coefficients of the provisions of the current joint venture

3. The resulting expert models should be recommended for use in order to increase the level of reliability of existing and planned transport facilities.

\section{References}

1. Yu.L. Vlasov, N.A. Morozov, A.A. Gavrilov, Bulletin of the Orenburg State University 9(158), 227-231 (2013)

2. C.V. Bespalko, T.G. Kurykina, V.I. Bogachev, A.A. Gerasimenko, Modern problems of railway transport (Russian University of Transport), 251-259 (2019)

3. I.V. Demyanushko, A.I. Starostin, Questions of structural mechanics and reliability of machines and structures (MADI), 16-26 (2012)

4. M.S. Vysotsky, Yu.M. Pleskachevsky, A.O. Shimanovsky, M.G. Kuznetsova, Mechanics of Machines, Mechanisms and Materials 3(20) - 4(21), 142-148 (2012)

5. A.O. Shimanovsky, M.G. Kuzniatsova, V.I. Yakubovich, International journal of mechanical engineering and robotics research 7(4), 438-443 (2018)

6. R.A. Ibrahim, Appl. Mech Rev. 2(54), 133-199 (2001)

7. I. Esen, International journal of mechanical sciences 153-154, 21-35 (2019)

8. J. Dai, M. Han, K.K. Ang, International journal of mechanical sciences 151, 85-94 (2019) 
9. L. An, D. Li, P. Tu, P. Yuan, Theoretical \& Applied mechanics letters 6, 186-194 (2016)

10. Ch. Liu, Z. Wang, Q. Gao, KSCE Journal of civil engineering 23(5), 2293-2302 (2019)

11. S.Yu. Gridnev, A.N. Budkova, Internet journal of Science of Science 7.6(31), 141 (2015)

12. A.O. Shimanovsky, Mechanics. Research and Teaching (Gomel) 5, 163-165 (2011)

13. S.Yu. Gridnev, A.N. Budkovoi, Y.I. Skalko, Advances in Intelligent Systems and Computing 983, 574-585 (2019)

14. S.Yu. Gridnev, A.N. Budkova, News of higher educational institutions. Technology of the textile industry 1(367), 198-206 (2017) 